\title{
Effects on pupil size of manual and verbal indicators of cognitive task fulfillment
}

\author{
HERB M. SIMPSON, CARLETON UNIVERSITY \\ ALLAN PAIVIO, UNIVERSITY OF WESTERN ONTARIO
}

A total of 20 Ss attempted to generate images to concrete and abstract stimulus words while pupillary activity was continuously photographed. One group $(\mathrm{N}=5)$ was required to press a key when an image occurred, a second to describe the image, and a third to press a key as well as describe the image. No overt indicator was required of a fourth group. Results showed that greater dilation occurred during the imagery task than during a control period only in those conditions in which an overt response indicated task fulfillment. Consistent with earlier findings significantly greater dilation occurred to abstract than concrete stimulus words in the key press condition.

The purpose of the present experiment was to investigate the effects on pupil size of overt motor responses typically used to indicate fulfillment of cognitive tasks. In a previous experiment (Paivio \& Simpson, 1966) pupillary dilation was shown to be associated with attempts to generate "mental images" to stimulus words, the amount of dilation being greater to abstract than concrete nouns. The increased pupil size during the imagery task as well as the greater dilation to abstract words was interpreted as indicating the difficulty of the cognitive task inasmuch as it has been shown elsewhere (e.g., Paivio, 1965) that it is more difficult to generate images to abstract terms. In the Paivio and Simpson experiment, Ss indicated fulfillment of the task (i.e., that they had a mental image) by pressing a telegraph key. It was found that the latency of the key press coincided with time to maximum pupil size, suggesting that the key press response contributed to the dilation. This possibility was investigated in a subsequent experiment (Simpson \& Paivio, 1966), which replicated the earlier study with the modification that no key press was involved. The amount of dilation during the imagery task was significantly reduced in the experiment without the key press, supporting the hypothesis that pressing the key contributed to the dilation.

The issue involved is of general importance because studies using pupil size as an index of mental activity (Beatty \& Kahneman, 1966; Hess \& Polt, 1964; Kahneman \& Beatty, 1966, 1967; Kahneman, Beatty, \& Pollack, 1967; Paivio \& Simpson, 1966) have typically involved an overt indicator of task fulfillment, and the contribution of such a response to the pupillary dilation, which is assumed to reflect the "mental" effort of the task, is indeterminate in these experiments. The most common indicators have been manual manipulation and verbal reports. Evidence of the effect of the former was provided by our previous research (Paivio \& Simpson,
1966; Simpson \& Paivio, 1966), while Hakerem and Sutton (1966) and Bernick and Overlander (1967) have recently shown that the pupillary response is also enhanced when verbalization is required of $\mathrm{S}$. The present experiment extended the prior research by including both classes of responses in one design. along with variation in the difficulty of a cognitive (imagery) task.

\section{HETHOD}

\section{Subjects}

Twenty volunteer students from introductory psychology classes at the University of Western Ontario served as Ss in the experiment. The Ss were randomly assigned to four groups of five Ss each, namely, a group that: pressed a key when an image had been generated and gave a description of the image (Group KD); only pressed a key (Group K); gave a description only (Group D); and neither pressed a key nor gave a description (Group NoKD).

\section{Stimuli}

The stimulus items were eight nouns, four abstract and four concrete, selected by the Es from a pool of items which had been rated on seven-point scales for abstractness-concreteness and the difficulty or ease with which the words evoke sensory images (Paivio, Yuille, \& Madigan, in press). The concrete words, which exceed the abstract in their rated capacity to evoke images, were coffee, house, pencil, and tree; the abstract terms were fate, moment, opinion, and virtue. The words were randomly assigned to each of two lists with the restriction that no more than two words of a type (i.e., concrete or abstract) occurred in sequence within a list. The lists were tape recorded, each word being separated by two signal tones (approximately $450 \mathrm{cps}$ at $50 \mathrm{~dB}$ ). Thus, the sequence of taped stimuli was: word, Tone 1 , Tone 2, word, Tone 1 , Tone 2, and so on, the interstimulus interval in each case being $10 \mathrm{sec}$.

\section{Apparatus}

The main part of the apparatus was essentially the same as that developed by Hess and Polt (see Hess, 1965, for illustrations). Basically, it was a $12 \times 12$ $x 24$ in. box with a goggle-like opening at one end, which supported S's face while he looked into the box. The other end of the box contained an $11 \times 11-1 / 2$ in. ground glass screen. A target in the form of a $1 \times 1 \mathrm{in}$. plus (t) sign located in the center of the screen was used as a fixation point for $S$. The screen was homo- 
geneously illuminated ${ }^{2}$ by a 12 in. fluorescent tube located outside the box 30 in. from the screen.

Photographs of S's right eye were taken by a $16 \mathrm{H}$ Bolex movie camera fitted with a $100 \mathrm{~mm}$ lens and 30 $\mathrm{mm}$ of extension tubes. The camera was mounted externally on the right side of the box and driven at a filming speed of two frames per second by a $15 \mathrm{rpm}$ motor. The lens of the camera protruded through an opening into the box directly in line with a $2 \times 3$ in. mirror, which reflected the image of S's right eye into the camera. Both the lens and mirror were below S's line of sight. The camera was loaded with Kodak high speed infrared film. A standard $25 \mathrm{~W}$ red bulb in a reflector approximately 8 in. from $S$ 's eye served as the infrared source. The combined illumination of the red bulb and external flourescent tube was approximately $40 \mathrm{ft}-\mathrm{c}$ at S's eye.

Stimuli were presented auditorially to $\mathbf{S}$ through earphones by means of a tape recorder. When a stimulus word was presented a clock started and was stopped when a telegraph key located on the table at the right of the box was pressed.

\section{Procedure}

The Ss were tested individually in a randomized block order, a block consisting of four Ss, one from each of the four groups. Half the Ss received List 1 of the stimulus items and half received List 2.

A separate set of tape recorded instructions was prepared for each group. The basic instructions were similar for all groups inasmuch as $\mathrm{S}$ was told to try to picture some object, event, or situation the word made him think about. The instructions differed among the groups, however, with respect to the key press and verbal description conditions. The Ss in the groups required to give a description (Groups $D$ and $K D$ ) were told to generate a mental image when they heard a stimulus word and to keep the image in their minds until they heard the first tone, which occurred 10 sec after the word and was their cue to begin a description of the mental image. The first tone was followed in $10 \mathrm{sec}$ by another tone, which was the signal for $S$ to stop the verbal description, if it were not already complete, and relax. The Ss in Groups $K$ and $K D$ were instructed to press the telegraph key as soon as they got a mental image, but to try to keep the image in their minds until they heard the first tone. For Group $\mathrm{KD}$ this was the cue to begin a description, but for Group $K$ it was the signal to recite the alphabet. The purpose of the alphabet recitation, which was used in Group NoKD as well as Group $K$, was to provide a control condition for the period of verbalization in Groups $D$ and $K D$. The Ss were told to recite the alphabet untll they heard the next tone, which was the cue to relax mentally.

The sequence of stimuli began with a tone signalling a "relax" period so that each 10 sec Image condition was preceded by a $10 \mathrm{sec}$ "control" period. Thus, the sequence of conditions was: relax, image, description or alphabet recitation, relax, image, and so on. In this

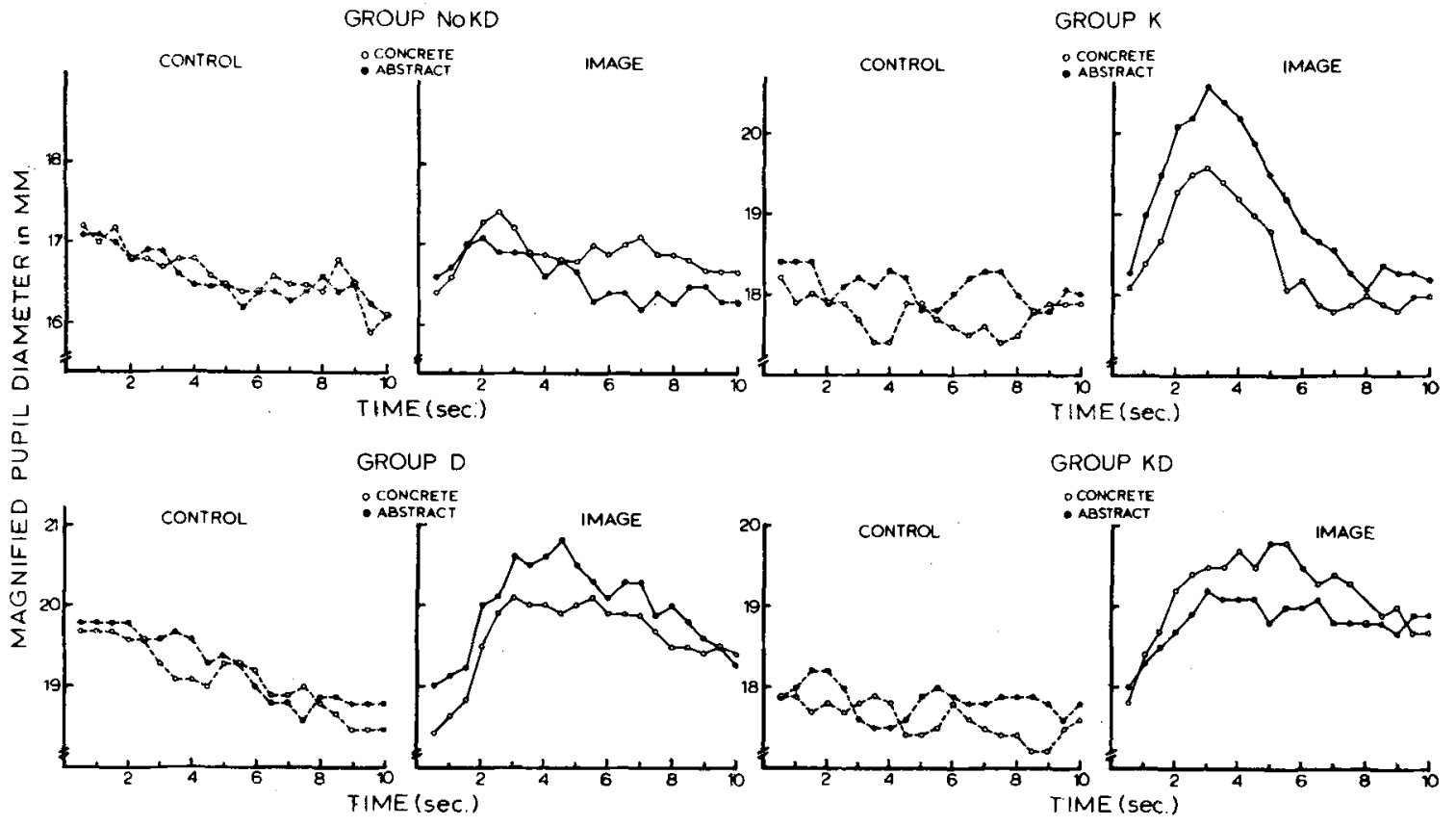

Fig. 1. Mean pupil diameter over time during control periods and while attempting to generate images to abstract and concrete words, for Ss instructed: to press a key when they had an image (group K); to give a description of the image following the image condition (group D); to both press the key and give a description (group KD); and for $\mathrm{Ss}$ required to do neither (group NoKD). 
way, an appropriate within Ss control was available for each image condition.

The processed movie film was examined frame by frame on a $4 \times 6$ in. film editor and the pupil diameter thus magnified was measured in millimeters. ${ }^{3}$

\section{RESULTS AND DISCUSSION}

For each photographic frame, pupil size was averaged across Ss, separately for concrete and abstract words as well as for control conditions ${ }^{4}$ preceding concrete and abstract stimuli. These data are presented for each group in Fig. 1. Each point in the figure is a mean based on 20 measurements (e.g., four abstract words for each of five Ss). Inspection of the curves for group NoKD indicates that pupil size during attempts to generate images was slightly larger than pupil size during the control periods. This effect was clearly more pronounced in Groups $\mathrm{K}, \mathrm{D}$, and $\mathrm{KD}$, however. In addition, the curves for Groups $K$ and $D$ show greater dilation during attempts to generate images to abstract than to concrete words. The opposite was the case for Groups NoKD and KD. The shapes of the curves during the image conditions also differ among the groups. For Group K, pupil size reaches a maximum early followed by rapid constriction, which continues until pupil size has reached the level similar to that preceding the imagery task. The dilation in Group $\mathrm{K}$ is quite transient, lasting approximately $7 \mathrm{sec}$ from the onset of the imagery task. Groups $\mathrm{D}$ and $\mathrm{KD}$ show the same initial dilation as Group $K$, but the pupil remains relatively dilated throughout the image condition in the groups involving a verbal description.

To evaluate the data statistically, mean scores for the various conditions were determined for $S$, averaging pupil size over the 20 photographic frames, separately for abstract and concrete stimuli for the image conditions, and the respective control conditions preceding abstract and concrete words. These data were

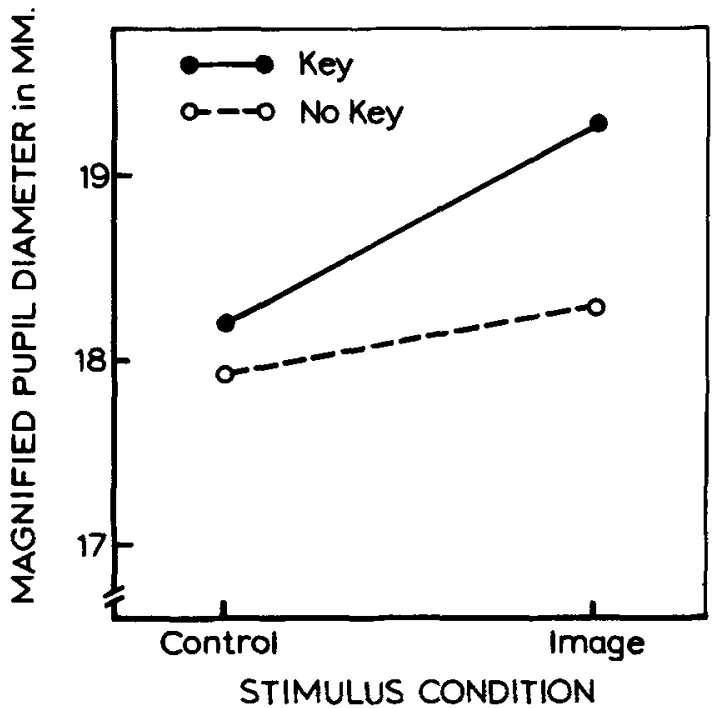

Fig. 2. Interaction effect of key press and stimulus conditions on pupil size. analyzed using a 2 by 2 by 2 by 2 analysis of variance with key (key press vs no key press), description (verbal description vs no verbal description), concreteness (abstract vs concrete stimuli), and stimulus conditions (control vs image periods) as variables. The last two involved repeated measures. The results showed a significant effect of conditions $(F=43.30$, df $=1 / 16, p<.01$ ), pupillary dilation being greater during image than control conditions. The interaction of key with stimulus conditions was also significant ( $F=9.13$, $\mathrm{df}=1 / 16, \mathrm{p}<.01$ ). This effect is plotted in Fig. 2, which shows that the difference in pupil size during image as compared with control conditions was greater when Ss pressed the telegraph key than when they did not. The corresponding interaction of description and stimulus conditions was not significant $(F=3.85$, $\mathrm{df}=$ 1/16). Also significant was the three-way interaction involving the variables key, description, and concreteness $(F=5.88, d f=1 / 16, p<.05)$, as well as the fourway interaction $(F=6.18$, $d f=1 / 16, p<.05)$. These effects were probably due largely to a reversal of the abstract-concrete difference, such that pupil size during the image period was greater to abstract than to concrete words in Groups $K$ and $D$, whereas the opposite was the case for Groups NoKD and KD (see Fig. 1).

In order to clarify the complex interactions involving conditions and concreteness, separate 2 by 2 analyses of variance were performed on the data for each group, with concreteness (abstract vs concrete stimuli) and stimulus conditions (control vs image periods) as the variables. For Group $K$, the main effect of stimulus conditions was significant $(F=26.01$, $d f=1 / 14, p<.01)$, indicating greater pupillary dilation during image than control conditions. The effect of concreteness would be revealed in an interaction involving stimulus conditions and concreteness, and this interaction, which is plotted in Fig. 3, was significant $(F=24.75$, $d f=1 / 4$,

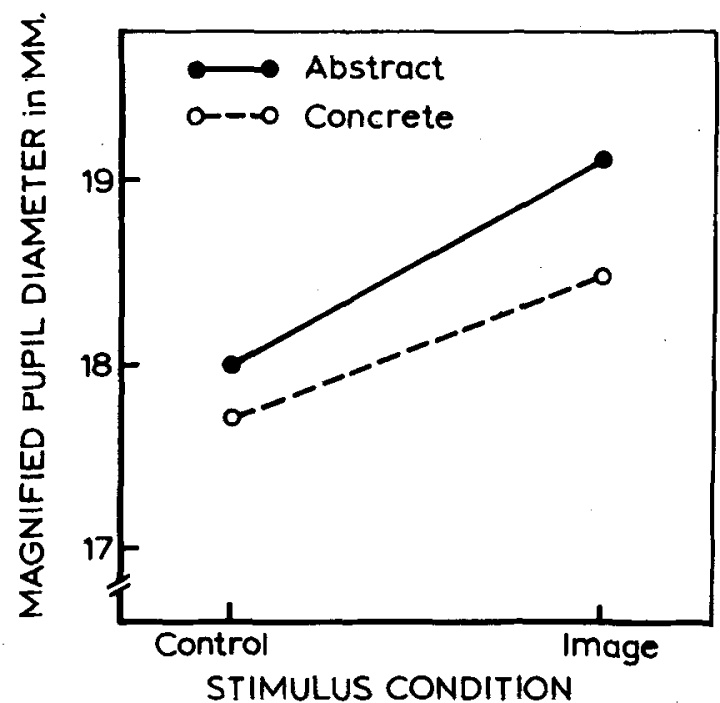

Fig. 3. Interaction effect of stimulus conditions and concreteness on pupil size for group $k$. 


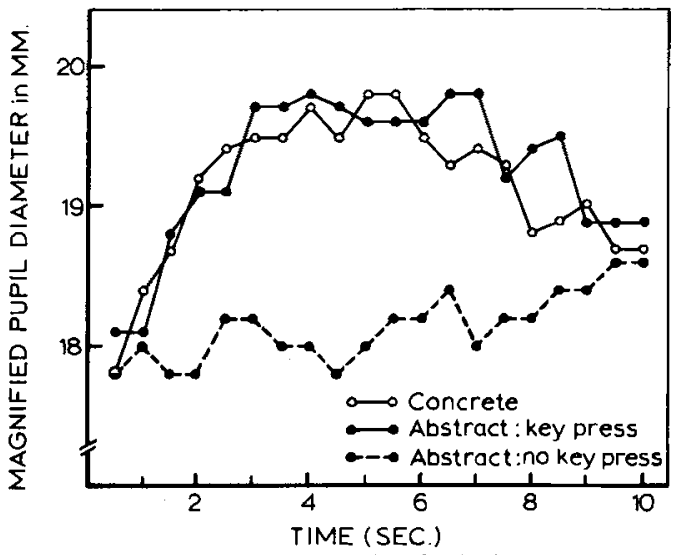

Fig. 4. Mean pupil diameter over time for Ss in group $\mathrm{KD}$ separating, for abstract words, those cases in which a key press occurred (upper curve) from those in which it did not (lower curve).

$\mathrm{p}<$.01). Relative to the corresponding control conditions, the dilation was greater during attempts to generate images to abstract than to concrete nouns. These results are entirely consistent with those of a previous experiment (Paivio \& Simpson, 1966), which involved essentially the same stimulus conditions as Group $\mathrm{K}$, indicating the reliability of the abstract-concrete difference when a key press response is used to indicate completion of the imagery task. No significant effects were obtained, however, for Group NoKD, supporting the visual inspection of the data for that group (see Fig. 1), which shows that pupil size during control and image conditions does not differ. These findings are also consistent with previous ones (Simpson \& Paivio, 1966), which showed that the amount of dilation during an imagery task was significantly reduced when no key press was included. For Group D, the main effect of conditions was significant $(F=8.51, d f=1 / 4$, $\mathrm{p}<.05)$, pupil size being greater during image than control periods. The interaction of conditions and concreteness was not significant $(F=0.70, d f=1 / 4)$, although the direction of the abstract-concrete difference (see Fig. 1) is consistent with the results for Group $K$ as well as those of the previous experiments (Paivio \& Simpson, 1966; Simpson \& Paivio, 1966). For Group $\mathrm{KD}$, the main effect of stimulus conditions as well as the interaction involving stimulus conditions and concreteness were significant $(F=39.50, \mathrm{df}=1 / 4$, $\mathrm{p}<.01$ and $F=11.49, \mathrm{df}=1 / 4, \mathrm{p}<.01$ ), respectively. As shown in Fig. 1, the concreteness effect in Group $\mathrm{KD}$ was the reverse of what has been consistently obtained, dilation being greater to concrete than abstract words in Group KD.

The unexpected results obtained in Group $\mathrm{KD}$ suggested that the reversal of the abstract-concrete difference was related to the different experimental conditions in that group. An examination of the key press data for Group $\mathrm{KD}$ revealed generally long reaction latencies to the abstract words and, in some cases, no latencies were recorded inasmuch as $S$ did not press the key within the $10 \mathrm{sec}$ period allotted.
Of the possible 20 instances (i.e., five Ss each responding to four abstract words) there were six cases, involving four of the five Ss, where no key press occurred. In effect, therefore, the intended experimental conditions for the group were not met on six occasions, since neither a key press nor the subsequent verbal description occurred. Consequently, the pupillary data for these cases were treated separately and appear for comparison purposes in Fig. 4, which shows the curves for the image condition of Group $\mathrm{KD}$. Each point in the upper and lower curves for the abstract words is based on 14 and six measurements, respectively, the lower one representing mean pupil size by photographic frame for the six cases where no key press and, therefore, no verbal description were obtained. Separating the data for those cases where the stimulus conditions were satisfied from those cases where they were not raised the curve for the abstract words involving a key press so that it is slightly higher than the curve for the concrete words. The curve based on the six conditions involving no key press is similar to the curves for the image condition of Group NoKD (see Fig. 1). This analysis, therefore, makes the data for Group KD less inconsistent with the abstractconcrete effect obtained in the other groups and provides further evidence that dilation during the imagery task is attenuated when no key press occurs.

\section{Key press latencies}

The latencies of key press responses to words were averaged across Ss separately for Groups $\mathrm{K}$ and $\mathrm{KD}$. $\mathrm{A}$ latency of $10 \mathrm{sec}$ was assigned to the six conditions of Group KD where no key press occurred. These data were analyzed using a 2 by 2 analysis of variance with concreteness (abstract and concrete words) and Groups ( $\mathrm{K}$ and $\mathrm{KD}$ ) as the variables. The means appear in Fig. 5. The main effect of concreteness was signifi-

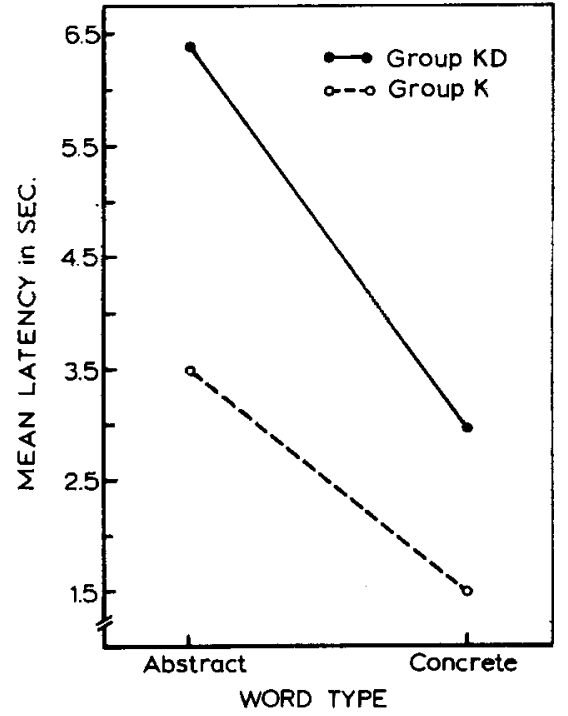

Fig. 5. Effect of requiring a description of the image (group $k$ versus KD) on key press latencies to abstract and concrete words. 
cant $(\mathrm{F}=83.37, \mathrm{df}=1 / 10, \mathrm{p}<.01)$, latencies being slower for abstract than concrete words. This finding is consistent with previous results (e.g., Paivio, 1966) and the interpretation that it is more difficult to generate images to abstract words (Paivio, 1965). The latencies for the $\mathrm{KD}$ group were also slower than those of the $K$ group $(F=11.19$, df $=1 / 10, p<.01)$, possibly reflecting an editing process. The $S$ may, for example, reject bizarre images or check to insure that he can give an adequate description of the image (Yuille, 1965): The interaction of concreteness with groups was not significant $(\mathrm{F}=3.29$, $\mathrm{df}=1 / 10)$.

\section{GENERAL DISCUSSION}

The results clearly show that pupillary dilation during an imagery task is enhanced when an overt motor response is required to indicate task fulfillment and the findings are, therefore, consistent with previous ones on the effect of a key press (Simpson \& Paivio, 1966) and of verbalization (Hakerem \& Sutton, 1966). The general significance of these findings is that the verbal or other responses typically used by investigators as indicators of task fulfillment (e.g., Hess \& Polt, 1964; Kahneman \& Beatty, 1966; Paivio \& Simpson, 1966) somehow contribute to the observed effects on pupil size. Why does the motor response have this effect? One possibility is that the requirement to make an overt response demands an explicit decision on the part of $S$, namely, whether the appropriate mental state (e.g., an image) is present or absent, and such a decision increases arousal level. A related possibility, which was also considered by Hakerem and Sutton (1966), is that increased activation results from motor feedback associated with anticipation of making the overt response, such as muscular tension in the hand, in the case of the key press, and covert verbalization, in the case of descriptions. Such an interpretation must be qualified by some characteristic of the anticipated response such as its relevance to the preceding cognitive task, inasmuch as dilation did not occur among Ss who were required simply to recite the alphabet following the imagery condition. Alternatively, S's arousal level is increased because the overt response (e.g., the description of an image) is publically observable and, therefore, exposes $S$ to evaluation (compare findings on audience effects, e.g., Paivio, 1965) and, in this context, Bernick and Oberlander (1967) suggested that the dilation observed during mental tasks may be a product of the emotion (anxiety) associated with making the subsequent response. Such an interpretation is complicated, however, by the data from Group $\mathrm{KD}$ of the present experiment. In that group the presumed anxiety-inducing requirement to respond was constant on all trials and, accordingly, dilation should have been observed in each case, but occurred only in those instances where $S$ did in fact carry out the set to respond (1.e.. he pressed the key). To make the anxiety notion tenable in this case it must be assumed that immediately upon presentation of a word $\mathrm{S}$ decided whether or not he would respond. The implication is that the observed dilation during mental tasks reflects the anxiety aroused by an early decision on the part of $S$ to make the appropriate overt response.

A final possibility is that feedback from the motor response itself contributes directly to the level of activation (cf. Nunnally, Knott, Kuchnowski, \& Parker, 1967), but here too the interpretation must be qualified by some characteristic of the response such as whether or not it indicates task completion. Hakerem and Sutton (1966) found dilation occurred when S pressed a switch to initiate a detection trial, but in the present experiment, where the key press indicated task fulfillment, pupil size was at a maximum prior to the occurrence of the key press. Similarly, Bernick and Oberlander (1967) showed that dilation occurred during verbalization of a problem, but a decrease in pupil size has typically been observed during or immediately following the verbal response that indicates task fulfillment (Beatty \& Kahneman, 1966; Hess \& Polt, 1964; Kahneman \& Beatty, 1966). The suggestions outlined above, of course, require independent testing.

Despite the complexities introduced by the contribution of the motor response to pupil size, there is still evidence to indicate that pupillary changes reflect the mental effort involved in cognitive tasks. Beatty and Kahneman (1966), for example, had Ss recall seven-digit telephone numbers from short and long term memory. Although the responses were similar in both tasks, greater dilation was associated with recall from long term memory supporting the authors' conclusion that the pupillary changes reflect the momentary state of mental effort (p. 372). Furthermore, it has been shown (Paivio \& Simpson, 1966, as well as Group $K$ of the present experiment) that the amount of dilation during an imagery task was significantly greater to abstract than to concrete words. The overt response was the same in all cases, namely, a key press, and the differential dilation to the abstract and concrete words may, therefore, reflect the relative complexity of the cognitive tasks. Nevertheless, the latency of the key press was longer to the abstract words, and it could be argued that the effect is somehow related to the latency of the overt response rather than to the cognitive difficulty of attempting to generate an image. The general problem merits further investigation using a variety of cognitive tasks, hopefully ones in which "mental effort" can be varied over a wide range without altering the parameters of the overt response, as well as ones in which mental effort remains constant while the response varies.

\section{References}

Beatty, J., \& Kahneman, D. Pupillary changes in two memory tasks. Psychon. Sci., 1966, 5, 371-372.

Bernick, N. \& Oberlander, M. Effect of yerbalization and two different rodes of experiencing on pupil size. Paper presented at the Fifth Colloquium on the Fupil, Philadelphia, May, 1967. 
Hakerem, G., \& Sutton, S. Pupillary response at visual threshold. Nature, 1966, 212, 485-486.

Hess, E. H. Attitude and pupil size. Scient. American, 1965 $212,46-54$.

Hess, E. H., \& Polt, J. M. Pupil size in relation to mental activity during simple problem-solving. Science, 1964, 143, 1190-1192.

Kahneman, D., \& Beatty, J. Pupil diameter and load on memory. Science, 1966, 154, 1583-1585.

Kahneman, D., \& Beatty, J. Pupillary responses in a pitch-discrimination task. Percept. \& Psychophys., 1967, 2, 101-105.

Kahneman, D., Beatty, J., \& Pollack, I. Perceptual deficit during a mental task. Science, 1967, 157, 218-219.

Nunnally, J. C., Knott, P. D., Duchnowski, A., \& Parker, R. Pupillary response as a general measure of activation. Percept. \& Psychophys., 1967, 2, 149-155.

Paivio, A. Personality and audience influence. In B. A. Maher (Ed.), Progress in experimental personality research, vol. 2 New York: Academic Press, 1965. Pp. 127-173.

Paivio, A. Abstractness, imagery, and meaningfulness in pairedassociates learning. J. verbal Leam. verbal Behav., 1965, 4 32-38.

Paivio, A. Latency of verbal associations and imagery to noun stimuli as a function of abstractness and generality. Canad. $J$. Psychol., 1966, 20, 378-387.

Paivio, A., \& Simpson, H. M. The effect of word abstractness and pleasantness on pupil size during an imagery task. Psychon. Sci., 1966, 5, 55-56.

Paivio, A., Yuille, J. C., \& Madigan, S. Concreteness, imagery and meaningfulness values for 925 nouns. J. exp. Psychol. Monogr. Suppl., in press.

Simpson, H. M., \& Paivio, A. Changes in pupil size during an imagery task without motor tesponse involvement. Psychon. Sci., 1966, 5, 405-406.

Yuille, J. C. Effects of noun imagery and meaning fulness on latencies of mediators and on learning. Unpublished M.A. thesis, University of Western Ontario, 1965.

\section{Notes}

1. This research was supported by grants APA-204 (to the first author) and APA-87 (to the second author) from the National Research Council of Canada as well as grants from the University of Western Ontario Research Fund. A paper based on this investigation was presented in a symposium on Pupillometric Research at the 1967 American Psychological Association meetings in Washington, D. C.

2. Photometric readings with a spot photometer (Photovolt Corp. Model 200) were taken at five places on the screen, namely, the four comers and the center. Recorded illumination at each of these places was approximately $15 \mathrm{ft}-\mathrm{c}$.

3. The authors gratefully acknowledge the assistance of Frank Colman with this portion of the experiment.

4. The data for pupil size during verbal descriptions of the image (groups D and KD) and during recitations of the alphabet (groups NoKD and $K$ ) are not presented. Descriptions were often accompanied with frequent eye blinks and eye movements yielding insufficient and/or contaminated (the pupil constricts to lid closure) data on which to base an analysis. In addition, for purposes of this experiment the crucial tests are based on comparisons between pupil size during attempts to generate images and a preceding control condition.

(Accepted for publication November $5,1967$. 